\title{
Comparaison de l'utilisation de sciure ou d'un mélange paille-sciure comme matériau de litière accumulée pour porcs charcutiers
}

\author{
Baudouin Nicks*, Alain Désiron, Bernard Canart \\ Université de Liège, Faculté de médecine vétérinaire, bld de Colonster, \\ $\mathrm{B} 43,4000$ Liège, Belgique
}

(Reçu le 18 août 1997; accepté le 28 novembre 1997)

\begin{abstract}
Comparison of two litter materials, sawdust and a straw-sawdust mixture, for fattening pigs on deep litter. Two similar rooms were used to keep pigs on deep litter, one room with pigs on sawdust (litter $S$ ) and the other with pigs on a mixture of straw and sawdust with $50 \%$ dry matter (DM) of each material (litter PS). Three batches of 17 pigs were reared in each room over the 14-month period of experiment. The deep litters were started with a bedding of about $30 \mathrm{~cm}$. Afterwards the amounts of litters used were adjusted to keep the pigs clean. Each week the litter S was manually aerated and manure incorporated in the litter. This work was not performed with the litter PS. Both temperatures, relative humidities and ventilation rates were similar in the two rooms. The temperatures in the litters were continuously recorded. Ammonia concentrations were measured once a week using 8-h diffusion tubes. Electrochemical probes were occasionally used to measure the $\mathrm{NH}_{3}$ concentrations continuously, at 1 -h intervals, during 1-week periods. NO concentrations were measured during all the experimental period with electrochemical probes. At the end of the experiment the amounts of litter DM used per pig were of $32 \mathrm{~kg}$ with the litter $S$ and $40 \mathrm{~kg}$ with the litter PS. The litter temperatures measured at $20 \mathrm{~cm}$ depth varied between 30 and $37^{\circ} \mathrm{C}$. Heat evaporated the dung water and the DM content of the composts produced staid above $50 \%$. The amounts of compost produced per fattening pig were of $127 \mathrm{~kg}$ with the litter $\mathrm{S}$ and of $157 \mathrm{~kg}$ with the litter PS. Corresponding amounts of $\mathrm{N}$ in the composts were of 1.38 and $1.92 \mathrm{~kg} /$ pig, respectively. $\mathrm{NH}_{3}$ emission was lower from litter $\mathrm{S}$ than from litter PS but small increases of $\mathrm{NH}_{3}$ concentration until $50 \mathrm{ppm}$ were observed during the work of aeration. Adding new litter decreased the emissions. NO emissions were only observed with the litter $\mathrm{S}$, during the aeration period. Pig performance and carcass qualities were not affected by the litter materials. (C) Elsevier / Inra)
\end{abstract}

deep litter / sawdust / compost / ammonia / fattening pig

* Correspondance et tirés à parts.

Tél. : 3243664145 ; fax : 3243664122 ; e-mail : nicksb@stat.fmv.ulg.ac.be 
Résumé - Deux litières de $30 \mathrm{~cm}$ d'épaisseur ont été constituées en vue de l'engraissement de trois lots de porcs sur chacune d'elles. Le matériau de litière était soit de la sciure (litière $S$ ) ou un mélange paille-sciure (litière PS) réalisé sur la base de $50 \%$ de matière sèche (MS) des deux constituants. En fonction de l'état de propreté des porcs, des apports supplémentaires de litières ont été réalisés au cours des 14 mois d'expérimentation. Les quantités de MS de litière utilisées par porc engraissé ont été de $32 \mathrm{~kg}$ avec la litière $S$ et de $40 \mathrm{~kg}$ avec la litière PS. Les températures des deux litières ont été comprises entre 30 et $37^{\circ} \mathrm{C}$, la teneur en MS du compost formé se maintenant au-dessus de $50 \%$. Les quantités de compost retirées en fin d'essai ont été de $127 \mathrm{~kg}$ par porc engraissé avec la litière $S$ et de $157 \mathrm{~kg}$ avec la litière $P S$ et les rejets d' $N$ dans les composts ont été respectivement de 1,38 et $1,92 \mathrm{~kg} / \mathrm{porc}$. La litière $S$ a dégagé moins d'ammoniac que la litière PS mais les opérations d'aération de la litière $S$ ont été accompagnées de dégagements de NO qui n'ont jamais été mis en évidence avec la litière PS. Les performances de croissance des animaux et la composition des carcasses ne différaient pas en fonction du type de litière. (@ Elsevier / Inra)

litière accumulée / sciure / compost / ammoniac / porcs charcutiers

\section{INTRODUCTION}

L'utilisation de sciure comme matériau de litière accumulée pour porcs charcutiers a été introduite en Europe à la fin des années 80 . Par rapport à la paille, la sciure offre l'avantage de permettre un travail régulier d'aération de la litière assurant un meilleur compostage des déjections. Les fumiers à base de paille, accumulés sous les porcs, présentent une structure trop compacte pour pouvoir être aérés. Ce sont dans ce cas des bactéries anaérobies qui assurent la transformation de la matière organique des déjections, avec pour conséquence une production plus importante d'ammoniac et de composés odorants [13]. Or, un des principaux avantages de l'utilisation de litières comparativement à la récolte des déjections sous forme de lisier, est la réduction de la nuisance olfactive $[3,8,11,16]$. On peut y ajouter une réduction de près de moitié du volume d'effluents produits $[9,18]$ et des conditions de logement plus favorables au bienêtre des porcs qui notamment peuvent extérioriser leur comportement fouisseur $[2,4]$.

Si la sciure apparaît comme un matériau plus intéressant que la paille pour obtenir un compostage in situ des déjections, elle est cependant loin d'être disponible partout en quantité suffisante pour pouvoir être utilisée à large échelle. C'est la raison pour laquelle une expérience a été mise en place pour comparer les évolutions d'une litière accumulée à base de sciure et d'une litière composée d'un mélange de paille et de sciure réalisé sur la base d'un apport d'une même quantité de matière sèche des deux matériaux.

Des mesures ont été réalisées durant 14 mois au cours desquels trois lots de porcs ont été hébergés sur chacune des deux litières, sans curage entre les lots. La composition des litières a été déterminée à intervalles réguliers et la température dans la masse, qui reflète l'activité bactérienne de décomposition de la matière organique, a été relevée en continu. La comparaison des émissions d'ammoniac s'est faite sur la base des relevés des concentrations de ce gaz dans l'air des locaux. Les performances des porcs ont été enregistrées.

\section{MATÉRIEL ET MÉTHODES}

\subsection{Description des locaux}

Deux locaux identiques et adjacents de $30,2 \mathrm{~m}^{2}$ et $106 \mathrm{~m}^{3}$ ont été utilisés pour l'hébergement de porcs sur litières accumulées, à base de sciure dans l'un et d'un mélange paillesciure dans l'autre. Dans chaque local une loge 
Tableau I. Importance pondérale de différentes classes de taille des particules de sciure.

\begin{tabular}{lcccccc}
\hline Classe de taille (mm) & $<1$ & $1-2$ & $2-4$ & $4-8$ & $8-16$ & $>16$ \\
Poids (\% du total) & 56 & 25 & 13 & 3 & 1 & 2 \\
\hline
\end{tabular}

a été aménagée pour 17 porcs à raison de' $1,2 \mathrm{~m}^{2}$ de surface de litière disponible par animal. La ventilation était assurée par un ventilateur réglé manuellement à l'aide d'un rhéostat. Le débit de ventilation a varié de $600 \mathrm{~m}^{3} / \mathrm{h}$ pour l'ensemble du local en début de période d'engraissement à $2000 \mathrm{~m}^{3} / \mathrm{h}$.local en fin de période. À tout moment le réglage était effectué de la même façon dans les deux locaux. L'air frais entrait dans les locaux après avoir été réchautfé au niveau d'un couloir d'accès.

\subsection{Réalisation et entretien des litières}

La sciure utilisée provenait d'un mélange d'essences de bois blancs. Elle était composée de particules de taille allant de moins de $1 \mathrm{~mm}$ à plus de $1,5 \mathrm{~cm}$ (tableau I). Les teneurs en matière sèche (MS) de la sciure et de la paille (de froment) étaient respectivement de 49 et $90 \%$.
La litière à base de sciure (litière $S$ ) a été aménagée avant l'arrivée des premiers porcs sur une épaisseur de $30 \mathrm{~cm}$ par apport de $147 \mathrm{~kg}$ de substrat par animal ( $72 \mathrm{~kg}$ de MS). Dans l'autre local, pour obtenir une épaisseur équivalente à partir d'un mélange paille-sciure (litière PS), $26 \mathrm{~kg}$ de paille et $48 \mathrm{~kg}$ de sciure, soit environ $23 \mathrm{~kg}$ de MS de chacun des deux substrats, ont été utilisés par animal. En fonction de l'état de propreté des porcs, des quantités supplémentaires de litières fraîches ont été ajoutées dans les deux loges. Ces apports supplémentaires ont débuté dix semaines après l'arrivée des premiers porcs dans la loge avec la litière PS et seulement au début de l'engraissement du troisième lot de porcs dans l'autre (figure 1). Après 14 mois de fonctionnement, les quantités totales de MS de litières utilisées par porc engraissé ont été respectivement de $32 \mathrm{~kg}$ de sciure et de $40 \mathrm{~kg}$ du mélange PS.

Contrairement à ce qui a été parfois préconisé, les litières n'ont pas été imprégnées de lisier et aucun additif n'a été ajouté ni au début ni en cours d'expérimentation.

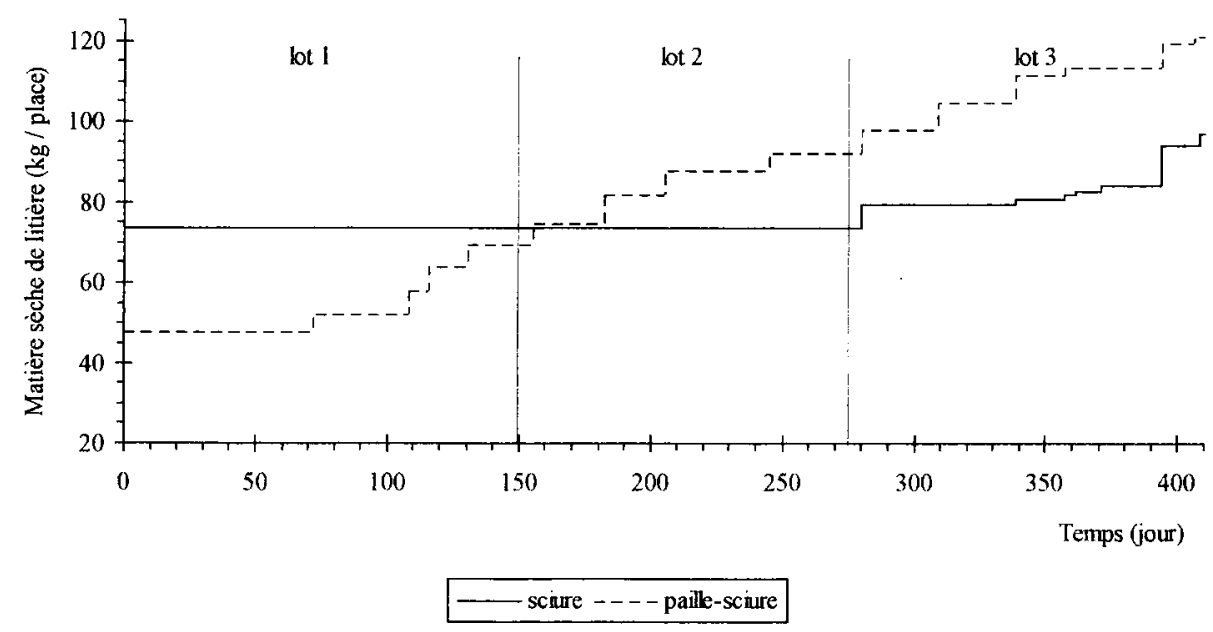

Figure 1. Évolution des quantités de matière sèche de litières utilisées par emplacement au cours des trois périodes d'engraissement. 
À raison d'environ une fois par semaine, les déjections des porcs élevés sur la litière de sciure étaient dispersées sur toute la superficie de la loge et enfouies dans la masse. Ces opérations, réalisées à l'aide d'une fourche, permettaient une aération régulière de la litière et une redispersion des fèces.

L'aspect de la litière PS était beaucoup plus proche de celui d'une litière de paille que de sciure, cette dernière ayant tendance à s'accumuler au niveau du sol. Dans ces circonstances, le travail de dispersion et d'enfouissement des déjections n'a pas été réalisé.

\subsection{Animaux et alimentation}

Au début de chaque période d'engraissement, 34 porcelets. tous issus du même élevage, étaient répartis en deux groupes homogènes sur la base d'un équilibre des poids et des sexes. Trois pertes accidentelles, une par période d'engraissement, sont survenues dans la loge avec la litière PS mais aucun problème sanitaire particulier n’a été observé durant les 14 mois d'expérimentation.

Les porcs, de type Landrace belge, étaient nourris ad libitum avec une farine du commerce à $20 \%$ de protéines brutes $(\mathrm{PB})$ au cours des deux premières semaines (aliment de transition) et à $18 \%$ de PB par la suite. L'équipement d'alimentation était du type bac à bouillie, avec tétine d'abreuvement au-dessus de la mangeoire.

Les vitesses de croissance des animaux ont été établies individuellement par pesées en début et fin d'engraissement, et les consommations d'eau et d'aliment ont été déterminées par lot.

\subsection{Mesures}

Dans chaque loge, quatre sondes de température ont été placées dans la litière à environ $20 \mathrm{~cm}$ de profondeur. Les mesures ont été réalisées à la cadence de une par heure et les résultats étaient stockés sur un enregistreur électronique (datalogger). Les températures et humidités relatives ambiantes ont également été relevées à la même fréquence.

Les concentrations en ammoniac ont été déterminées à l'aide de tubes réactifs à diffusion (Gastec) à raison d'un relevé par semaine.
Ces tubes fournissent la concentration moyenne observée sur une période déterminée, soit lors de cette expérience, de $9 \mathrm{~h}$ à $17 \mathrm{~h}$. Cette technique de mesure, simple et peu coûteuse, offre la précision nécessaire pour établir des comparaisons entre des concentrations relevées dans diverses conditions. En effet, dans ce cas, on s'intéresse plus aux différences observées qu'aux valeurs absolues. C'est en ces termes que nos résultats ont été interprétés. Une détermination précise des émissions d $\mathrm{d}^{\circ} \mathrm{NH}_{3}$ à partir des litières aurait nécessité des appareillages plus sophistiqués [1] relevant les concentrations en continu durant toute la période d'expérimentation. Des mesures complémentaires ont bien été effectuées en continu, mais occasionnellement, avec des sondes à mesure électrochimique (Dräger), à raison d'un relevé par heure durant une semaine après quoi les sondes étaient recalibrées. Les concentrations en NO ont été mesurées en permanence avec un capteur électrochimique Dräger.

Des échantillons de litière ont été prélevés à cing reprises pour suivre l'évolution de la composition. La teneur en $\mathrm{N}$ des composts a été déterminée par la méthode Kjeldahl et la teneur en $\mathrm{C}$ a été calculée sur base de 55,55\% de $\mathrm{C}$ dans la matière organique [18].

Les analyses statistiques de comparaison de deux moyennes ont été effectuées en utilisant le test $t$ de Student.

\section{RÉSULTATS}

\section{1. Évolution des températures des litières}

Les températures ont évolué de façon comparable dans les deux litières. Après l'arrivée des premiers porcelets, elles ont augmenté progressivement de 18 à $30^{\circ} \mathrm{C}$ en l'espace d'une vingtaine de jours. Les températures moyennes ont été respectivement de $32,6^{\circ} \mathrm{C}$ avec la litière $\mathrm{S}$ et de $33,8{ }^{\circ} \mathrm{C}$ avec la litière PS. Des pointes atteignant $50^{\circ} \mathrm{C}$ ont été observées dans les deux cas. De même, les températures et humidités relatives de l'air ont quasi été maintenues identiques dans les deux locaux au cours de l'engraissement des trois lots de porcs (tableau II). 
Tableau II. Températures des litières et caractéristiques climatiques des locaux au cours des trois périodes d'engraissement.

\begin{tabular}{|c|c|c|c|c|c|c|}
\hline \multirow[t]{3}{*}{ Type de litière } & \multicolumn{3}{|c|}{ Sciure } & \multicolumn{3}{|c|}{ Paille-sciure } \\
\hline & \multicolumn{3}{|c|}{ périodes } & \multicolumn{3}{|c|}{ périodes } \\
\hline & 1 & 2 & 3 & 1 & 2 & 3 \\
\hline \multirow{3}{*}{$\begin{array}{l}\text { Température } \\
\text { de litière } \\
\text { Température } \\
\text { ambiante } \\
\text { Humidité relative } \\
\text { ambiante }\end{array}$} & $31,6 \pm 6,7$ & $33,8 \pm 4,6$ & $32,5 \pm 5,2$ & $29,8 \pm 7,4$ & $36,8 \pm 6,7$ & $34,8 \pm 5,2$ \\
\hline & $19,1 \pm 2,3$ & $21,6 \pm 2,4$ & $19,9 \pm 2,3$ & $18,4 \pm 2,3$ & $21,2 \pm 2,4$ & $18,4 \pm 2,5$ \\
\hline & $45,0 \pm 7,3$ & $63,6 \pm 8,4$ & $56,7 \pm 6,9$ & $46,6 \pm 7,3$ & $65,7 \pm 8,6$ & $58,9 \pm 7,3$ \\
\hline
\end{tabular}

\subsection{Quantités de litières récoltées et composition}

Les poids de litières enlevées après l'engraissement successif de trois lots de porcs ont été respectivement de $127 \mathrm{~kg} /$ porc pour la litière $S$ et de $157 \mathrm{~kg} /$ porc pour la litière PS. Ces quantités de litière contenaient respectivement 1,38 et $1,92 \mathrm{~kg}$ d'azote.

Le tableau III donne la composition des deux composts en fin d'essai. La teneur en MS de la litière $S$ a augmenté au cours des quatre premiers mois d'occupation de la loge, passant de 49 à $59 \%$. Ensuite, elle a diminué, mais après 14 mois d'utilisation elle était encore supérieure à la teneur en MS de la sciure fraîche (52 vs $49 \%$ ). Quant à la teneur en MS de la litière PS, elle est passée de 63 à $49 \%$, du début à la fín de l'essai.

Le rapport carbone/azote a progressivement diminué, passant de 82 à 21 avec la litière $S$ et de 80 à 18 avec la litière PS.

\subsection{Concentration en ammoniac dans l'air des locaux}

La figure 2 donne les concentrations moyennes en $\mathrm{NH}_{3}$ observées dans les deux locaux au cours des trois périodes
Tableau III. Caractéristiques du compost à base de sciure ( $\mathrm{S}$ ) et du mélange paille-sciure (PS) en fin d'essai.

\begin{tabular}{lcc}
\hline Type de compost & $\mathrm{S}$ & $\mathrm{PS}$ \\
\hline Matière sèche $(\%)$ & 52,1 & 49,2 \\
$\mathrm{~N}$ total $(\mathrm{g} / \mathrm{kg} \mathrm{MS})$ & 20,7 & 24,8 \\
$\mathrm{~N}$ ammoniacal $(\mathrm{g} / \mathrm{kg} \mathrm{MS})$ & 2,0 & 2,3 \\
$\mathrm{P}_{2} \mathrm{O}_{5}$ (g/kg MS) & 32,6 & 29,5 \\
$\mathrm{~K}_{2} \mathrm{O}$ (g/kg MS) & 42,8 & 41,0 \\
$\mathrm{C} / \mathrm{N}$ & 20,6 & 17,7 \\
\hline
\end{tabular}

d'engraissement. En deuxième période la concentration moyenne a été significativement plus faible $(P<0,001)$ avec la litière $S$ (16 vs $21 \mathrm{ppm}$ ). Si la ventilation était réglée en permanence de la même façon dans les deux locaux, les débits ont varié dans le temps en fonction de la température de l'air prélevé dans le couloir d'accès. Celle-ci fut en moyenne de $17,4{ }^{\circ} \mathrm{C}, 21,5^{\circ} \mathrm{C}$ et $17,1^{\circ} \mathrm{C}$ au cours des trois périodes. On peut donc considérer que, globalement, les débits ont été presque identiques en périodes 1 et 3 , le niveau de ventilation ayant dû être un peu plus élevé lors de la deuxième. La figure 3 montre les fluctuations journalières relevées durant $7 \mathrm{j}$ de mesures en continu, en fin de la première période d'engraisse- 


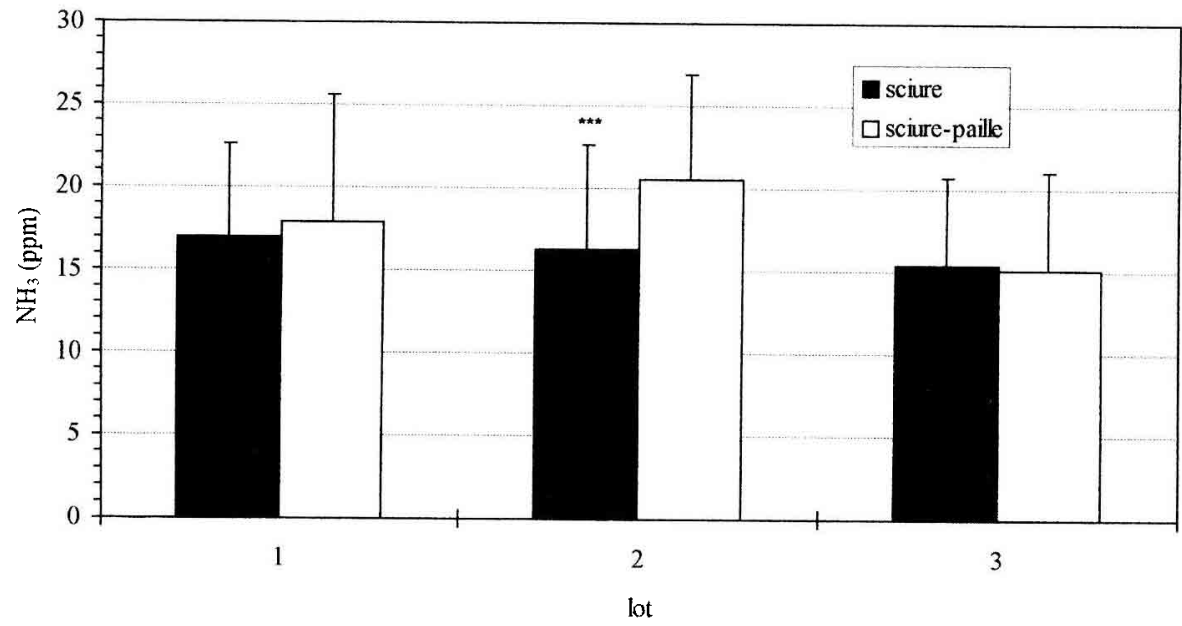

Figure 2. Concentrations moyennes en ammoniac dans l'air des deux locaux au cours des trois périodes d'engraissement.

ment. Dans les deux locaux, la concentration était minimale en période nocturne (de minuit à environ $6 \mathrm{~h}$ ) et maximale l'après-midi. Dans le local avec la litière PS, l'apport de litière fraîche $(5 \mathrm{~kg}$ de sciure et $2,65 \mathrm{~kg}$ de paille au $\mathrm{m}^{2}$ ) a entraîné une brusque diminution de la concentration en $\mathrm{NH}_{3}$, dont les effets ne se sont estompés qu'après environ $24 \mathrm{~h}$.

\subsection{Performances des animaux}

Les performances zootechniques moyennes sont rapportées dans le tableau $I V$. Aucune différence significative n'a été observée en fonction du type de litière pour la vitesse de croissance et la qualité de carcasse. Cette dernière a été objectivée par les prix moyens au kilo de carcasse payés en fonction de la qualité. La différence de prix moyen était inférieure à $1 \%$.

\section{DISCUSSION}

La façon la plus pratique de juger du bon fonctionnement d'une litière com- postée est d'en suivre la température. Nos résultats montrent que des températures supérieures à $30^{\circ} \mathrm{C}$, mesurées à $20 \mathrm{~cm}$ sous la surface, sont suffisantes pour empêcher l'accumulation d'eau dans la litière, ce qui confirme les observations de Nicks et al. [13] et de Kaufmann [11].

Les quantités de MS de litières utilisées correspondent à la limite inférieure de ce qui est habituellement recommandé pour les litières accumulées $[11,13,17]$. Sur la base d'un prix d'achat de $0,2 \mathrm{~F}$ le kilo de sciure fraîche livrée et de $0,42 \mathrm{~F}$ le kilo de paille, les coûts de litières par porc ont été de 13,4 et 17,6 F respectivement pour les litières $S$ et $P S$. Ces prix sont évidemment à calculer au cas par cas en fonction du lieu et du moment.

Dans les porcheries où les déjections sont récoltées sous forme de lisier, la production d'effluent par porc engraissé est estimée à $0,45 \mathrm{~m}^{3}$ ou $465 \mathrm{~kg}$ [19]. La technique de la litière accumulée a permis, dans cet essai, de réduire la masse d'effluent de $73 \%$ avec la litière $S(127 \mathrm{~kg}$ de compost/porc) et de $68 \%$ avec la litière PS (157 kg de compost/porc). Le taux 
litiere $S$

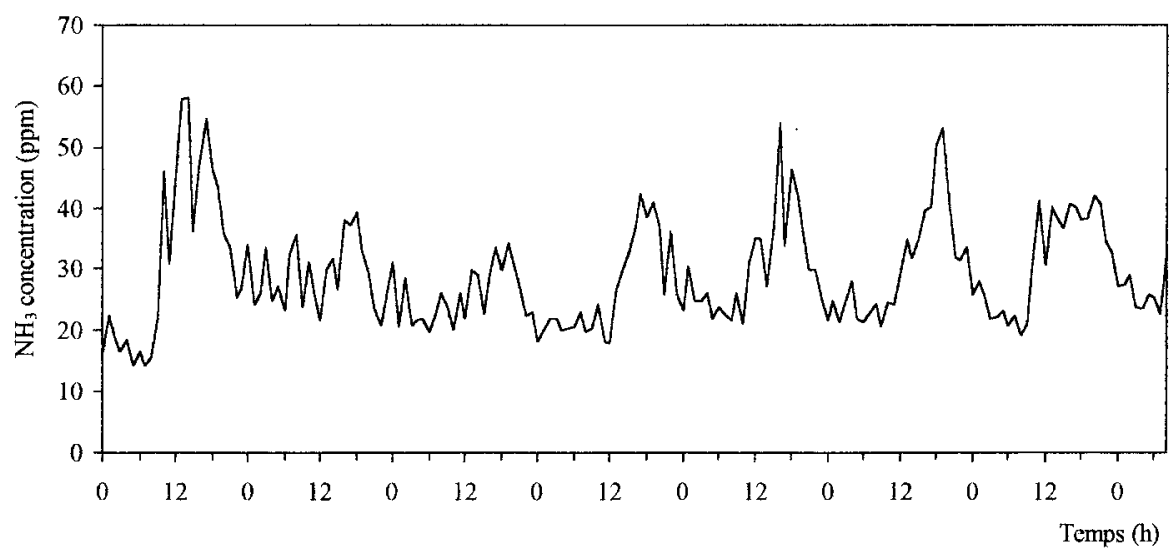

litière PS

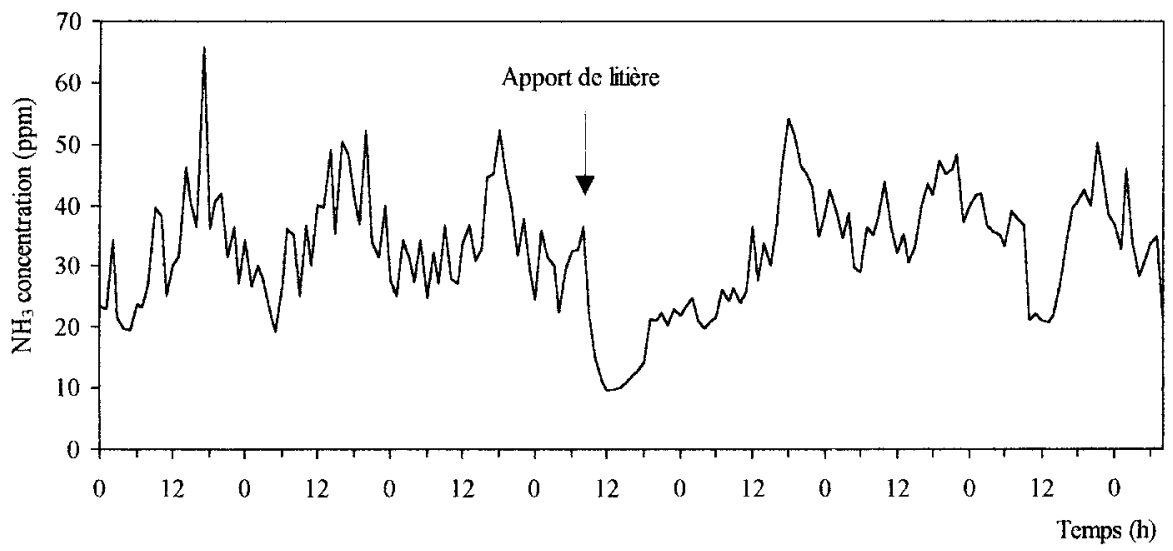

Figure 3. Évolution de la concentration en $\mathrm{NH}_{3}$ dans l'air des deux locaux au cours d'une semaine de relevés en continu.

d'évaporation de l'eau des composts a été de $85 \%$ avec la litière $\mathrm{S}$ et de $79 \%$ avec la litière PS. Ces valeurs ont été obtenues en divisant la quantité d'eau présente dans les composts à la fin de l'expérimentation par la somme des quantités apportées par les litières et les déjections des porcs. L'eau excrétée par les déjections a été estimée sur la base de $3 \mathrm{~kg}$ par porc et par jour [12]. Ces auteurs rapportent un taux d'évaporation de $75 \%$ à partir d'un compost à base de sciure produit à raison de $220 \mathrm{~kg}$ par porc engraissé.

En se basant sur des rejets d' $\mathrm{N}$ dans le lisier de 3,4 kg par porc engraissé [6] on peut calculer que les quantités d'azote présentes dans les composts en fin d'essai, soit $1,38 \mathrm{~kg} \mathrm{~d}$ 'N/porc avec la litière $\mathrm{S}$ et $1,92 \mathrm{~kg} /$ porc avec la litière PS, étaient infé- 
Tableau IV. Performances zootechniques.

Type de litière

\begin{tabular}{lcc}
\hline & Sciure & Paille-sciure \\
& $(n=51)$ & $(n=48)$ \\
Poids initial $(\mathrm{kg})^{*}$ & $22,8 \pm 2,9$ & $23,0 \pm 3,0$ \\
Poids final $(\mathrm{kg})^{*}$ & $109 \pm 11$ & $112 \pm 11$ \\
GMQ $(\mathrm{g})^{*}$ & $718 \pm 79$ & $746 \pm 74$ \\
Durée engraissement (jour) & 120 & 120 \\
Aliment consommé $(\mathrm{kg} / \mathrm{j})$ & 2,168 & 2,170 \\
Eau bue (L/kg d'aliment) & 2,06 & 2,08 \\
Indice de consommation $(\mathrm{kg} / \mathrm{kg})$ & 3,02 & 2,91
\end{tabular}

(*) moyenne et écart-type, les différences en fonction du type de litière ne sont pas significatives $(P>0,05)$

rieures de $59 \%$ et de $44 \%$ à celle obtenue quand les déjections sont récoltées sous forme de lisier. Une réduction de $73 \%$ $(0,88 \mathrm{~kg}$ d'N de compost/porc) a été signalée par Lesguillier et al. [12]. Kaufmann [11] rapporte une production de $0,71 \mathrm{~kg}$ d' $\mathrm{N}$ de compost/porc pour la période allant des poids moyens de 45 à $95 \mathrm{~kg}$. L'ensemble de ces données signifie que les pertes par volatilisation à l'intérieur des porcheries sont plus importantes avec la litière accumulée comparativement au lisier. En tenant compte des apports d'N alimentaire et de litières, des teneurs dans les composts et de l'azote fixé par les animaux, les pertes par volatilisation ont été estimées à $52 \%$ avec la litière $\mathrm{S}$ et à $45 \%$ avec la litière PS.

Les pertes d' $\mathrm{N}$ par volatilisation correspondent à des émissions d'ammoniac, d'oxydes d'azote et d'azote $\left(\mathrm{N}_{2}\right)$. Nos mesures n'ont pas permis d'estimer les parts respectives de ces trois formes azotées. Sur la base des relevés de concentrations on peut cependant dire que les émissions d' $\mathrm{NH}_{3}$ à partir des deux litières étaient équivalentes en première et troisième périodes d'engraissement tout en ayant été plus élevées avec la litière PS en deuxième période. Les estimations d'émission d' $\mathrm{NH}_{3}$ à partir de litières compostées sont peu nombreuses, variant de
1,1 à $2,9 \mathrm{~kg} /$ place par an $[15,18]$. Cette variation peut s'expliquer par le grand nombre de facteurs susceptibles d'interférer avec les émissions : caractéristiques de la litière, fréquence et mode d'aération, rapport $\mathrm{C} / \mathrm{N}$, activité des porcs, etc. Nicks et al. [13] avaient obtenu une émission plus élevée de $68 \%$ avec une litière de paille comparativement à une litière de sciure. Cette information, combinée aux résultats de cet essai, suggère que la litière PS s'est comportée plus comme une litière de sciure que comme une litière de paille. Kaiser et Van den Weghe [10] ont également observé des différences d'émissions entre deux litières, l'une à base de sciure, l'autre à base de paille. Dans le premier cas, les rejets d' $\mathrm{N}$ dans l'atmosphère se faisaient sous forme de $\mathrm{N}_{2}, \mathrm{NH}_{3}$ et $\mathrm{N}_{2} \mathrm{O}$ à raison de $78 \%, 12 \%$ et $10 \%$ respectivement ; dans le second cas, ils se faisaient sous forme de $\mathrm{N}_{2}$ et d' $\mathrm{NH}_{3}$ à raison de $63 \%$ et $27 \%$. À partir des lisiers, les émissions d'oxydes d'azote sont inexistantes et, bien qu'ils rejettent plus d' $\mathrm{NH}_{3}$ que les litières à base de sciure, ils émettraient globalement moins d' $N$ sous forme de composés polluants [7]. Les émissions d'N sous forme d' $\mathrm{NH}_{3}$ et de $\mathrm{NOx}$ à partir des litières présentent cependant une grande variabilité, presque du simple au double selon les mesures effectuées par Groenestein et Van Fassen [7]. Pour obte- 
nir un bon compostage, le rapport $\mathrm{C} / \mathrm{N}$ doit être compris entre 25 et 35 [5]. Or, ce rapport est en continuelle évolution au niveau des litières accumulées, passant de valeurs largement supérieures à 35 en début d'utilisation à des valeurs inférieures à 25 après 4 à 6 mois. Au-delà de cette période il serait possible de maintenir le rapport dans les limites souhaitées en ajoutant plus de litière mais la prise en compte des coûts de production vise à en utiliser le moins possible. Il y a donc ici opposition d'intérêts entre les aspects financiers et environnementaux.

Concernant les performances zootechniques et la qualité de la carcasse des porcs, nos données confïrment des résultats antérieurs prouvant que l'élevage sur litière permet d'obtenir des résultats au moins aussi bons que ceux réalisés par des porcs élevés sur caillebotis [14].

\section{CONCLUSION}

L'élevage de pores sur litière accumulée offre comme avantages au niveau environnemental : une forte réduction de la quantité d'effluents produits, une épuration directe de l'eau des déjections restituée sous forme de vapeur et une diminution de la pression azotée dans les plans d'épandage. Bien que la nuisance olfactive n'ait pas été directement mesurée dans cet essai, on peut cependant ajouter que le niveau d'odeur était très faible. Il reste cependant à mieux connaître les formes de rejets d' $\mathrm{N}$ sous forme gazeuse et des études devraient être poursuivies pour déterminer les conditions optimales privilégiant la formation de $\mathrm{N}_{2}$. Par rapport au lisier, la litière accumulée serait pénalisée si son utilisation s'accompagnait d'un accroissement des émissions d' $\mathrm{NH}_{3}$ et d'oxydes d'azote. Pour établir un bilan environnemental global il conviendrait également de disposer de données détaillées sur les possibilités d'utilisation agronomique du compost produit.

L'utilisation d'un mélange paille-sciure comme substrat de litière consomme une plus grande quantité de MS de litière que l'emploi de sciure seule et aboutit à la production d'une plus grande quantité de compost. Le devenir de l'azote des déjections est différent selon les substrats. La litière de sciure conserve moins d'azote, une plus grande quantité étant éliminée sous forme gazeuse avec notamment une production d'oxyde d'azote qui ne s'observe pas avec le mélange paillesciure.

\section{RÉFÉRENCES}

[1] Amon B., Boxberger J., Amon Th., Gronauer A., Depta G., Neser S., Schäfer K., Methods for measuring emissions from agrarian sources: FTIR measurement techniques with white-cell, large chamber or open-path, in: Procecdings of the International Symposium Ammonia and Odour Control from Animal Production Facilities, Vinkeloord, The Netherlands, October 6-10, 1997. pp. 161-167.

[2] Böhmer M.. Hoy S., Keeping fattening pigs on deep litter system with additives for fermentation in comparison with slatted floor system from the ethological point of view, in: Proceedings of the 8 th International Congress on Animal Hygiene, St. Patul, Minnesota, USA, 1994, pp. 29-32.

[3] Bonazzi G.. Navarotto P.L., Wood shaving litter for growing-finishing pigs, in: Voermans J.A.M. (Ed.), Proceedings workshop deep litter systems for pig farming, Rosmalen, 21-22 September 1992, Research Institute for Pig Husbandry, Rosmalen, The Netherlands, 1992, 57-76.

[4] Bruce J.M., Straw-Flow: a high welfare system for pigs. Farm Build. Progr. 102 (1990) 9-13.

[5] Forshell L.P.. Composting of catte and pig manure in Sweden, in: Ekesbo I (Ed.), Environment and animal health, Proceedings of the sixth International Congress on Animal Hygiene, 1988, 2, 650-657.

[6] Granier R., Texier C., Production de lisier du porc à l'engrais: quantité et qualité, TechniPorc 16.3.93 (1993) 23-31.

17) Groenestein C.M., Van Faassen H.G., Volatilization of ammonia, nitrous oxide and nitric 
oxide in deep-litter systems for fattening pigs, J. Agric. Eng. Res. 65 (1996) 269-274.

[8] Hesse D., Straw in fattening pig husbandry, in: Voermans J.A.M. (Ed.), Proceedings workshop deep litter systems for pig farming, Rosmalen, 21-22 September 1992, Research Institute for Pig Husbandry, Rosmalen, The Netherlands, 1992, pp. 77-92.

[9] Huysmans C.N., Greutink G.J., Onderzoek aan een diepstrooiselsystemen op praktijkbedrijven, in: Proefverslag P1.91, Ed.: Proefstation voor de Varkenshouderij, Postbus 83, 5240 AB Rosmalen, The Netherlands, 1993 , $92 \mathrm{p}$.

[10] Kaiser S., Van den Weghe H., Regulatory control of nitrogen emissions in a modified deep litter system, in: Proceedings of the International Symposium Ammonia and Odour Control from Animal Production Facilities, Vinkeloord, The Netherlands, October 6-10, 1997, pp. 667-675.

[11] Kaufmann R., Litière biomaîtrisée pour porcs à l'engrais: amélioration de la technique et valorisation de données importantes pour l'environnement, in: $29^{e}$ Journées de la recherche porcine en France, Paris, 4-6 février 1997, Institut technique du porc, Paris, 29, 1997, 311-318.

[12] Lesguillier F., Gouin R., Guiziou F., Orain B., L'élevage de porcs sur litières biomaîtrisées: contribution au dossier environnemental par l'évaluation des rejets, bilans des éléments azotés et minéraux des litières, in: $27^{\mathrm{e}}$ Journées de la recherche porcine en France, Paris, 31 janvier et 1-2 février 1995, Institut technique du porc, Paris, 27, 1995, 343-350.

[13] Nicks B., Désiron A., Canart B., Bilan environnemental et zootechnique de l'engraissement de quatre lots de porcs sur litière bio- maîtrisée, in: $27^{\mathrm{e}}$ Journées de la recherche porcine en France, Paris, 31 janvier et 1-2 février 1995, Institut technique du porc, Paris, 27, 1995, 337-342.

[14] Nicks B., Désiron A., Canart B., Clinquart A., Comparaison des performances de porcs élevés sur litières ou sur caillebotis, Ann. Méd. Vét. 140 (1996) 445-450.

[15] Oosthoek J., Milieuaspecten bij diepstrooiselsystemen voor vleesvarkens, in: Voermans J.A.M. (Ed.), Inleidingen Studiemiddag diepstrooiselsysteemen, Proefstation voor de Varkenshouderij, Postbus 83, 5240 AB Rosmalen, The Netherlands, (1993) pp. 17-23.

[16] Shilton A., Shallow beds, mean simpler waste management, Pig Int. August (1994) 15-16.

[17] Texier C., Vaudelet J.C., Rousseau P., Les porcheries sur litière accumulée: température, composition et volume des litières observés dans dix élevages, in: $29^{\mathrm{e}}$ Journées de la recherche porcine en France, Paris, 4-6 février 1997, Institut technique du porc, Paris, 29 (1997) 305-310.

[18] Thelosen J.G.M., Van Cuyck J.H.M., Voermans J.A.M., Vergelijking diepstrooiselsystemen met een traditioneel huisvestingssysteem; praktische ervaringen, in: Proefverslag P1.106, maart. Ed.: Proefstation voor de Varkenshouderij, Lunerkampweg 7, 5245 NB Rosmalen, The Netherlands, 1994, 78 p.

[19] Thelosen J.G.M., Voermans J.A.M., Comparison of two deep litter systems for fattening pigs with a traditional housing system; influence on some environmental aspects, in: Voermans J.A.M. (Ed.), Proceedings workshop deep litter systems for pig farming, Rosmalen, 21-22 September 1992, Research Institute for Pig Husbandry, Rosmalen, The Netherlands, 1992, pp. 26-35. 